
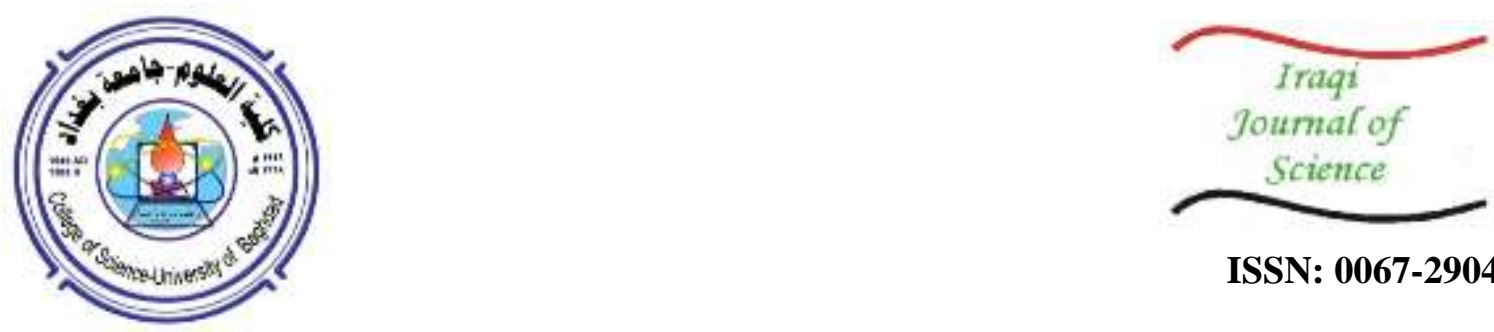

ISSN: 0067-2904

\title{
Learning Evolution: a Survey
}

\author{
Nada HussainAli ${ }^{* 1}$, Matheel Emaduldeen Abdulmunem², Akbas Ezaldeen Ali $^{2}$ \\ ${ }^{1}$ Imam Ja'afar Al-Sadiq University \\ ${ }^{2}$ Computer Science Department, University of Technology, Baghdad, Iraq
}

Received: 18/11/2020

Accepted: $18 / 2 / 2021$

\begin{abstract}
:
Learning is the process of gaining knowledge and implementing this knowledge on behavior. The concept of learning is not strict to just human being, it expanded to include machine also. Now the machines can behave based on the gained knowledge learned from the environment. The learning process is evolving in both human and machine, to keep up with the technology in the world, the human learning evolved into micro-learning and the machine learning evolved to deep learning. In this paper, the evolution of learning is discussed as a formal survey accomplished with the foundation of machine learning and its evolved version of learning which is deep learning and micro-learning as a new learning technology can be implemented on human and machine learning. A procedural comparison is achieved to declare the purpose of this survey, also a related discussion integrates the aim of this study. Finally a concluded points are illustrated as outcome which summarized the practical evolution intervals of the machine learning different concepts.
\end{abstract}

Keywords: Learning, Machine Learning, Deep Learning, Micro-learning.

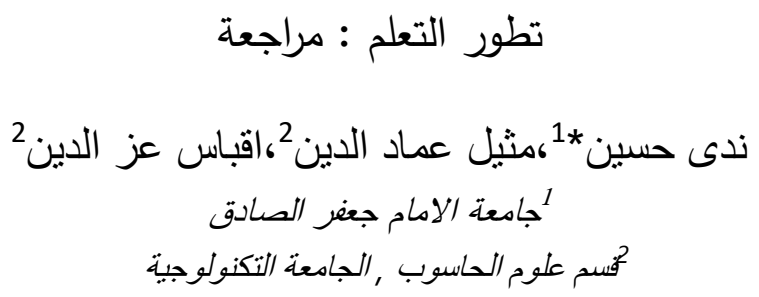

الخلاصه

التعلم هو عملية اكتساب المعرفة وتطبيق هذه المعرفة على السلوك. مبدأ التعلم غير محدود بالانسان

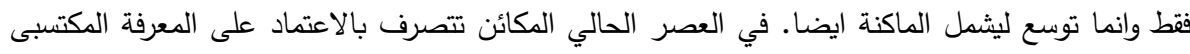

من البيئه.عملية التعلم تطورت في كلا الاتجاهين (الانسان والماكنة) لمواكبة التطور التكنولوجي الحاصل في لئي

العالم.تعلم الانسات تطور الى التعلم الدقيق وتعلم الماكنة تطور الى التعلم العميق. في هذا البحث يتم مناقثة

تتطور التعلم من اساسيات تعلم الماكنة والنسخة المطورة التعلم العميق والتعلم الدقيق كتقنية جديدة ممكن ان

تطبق على تعلم الانسان وتعلم الماكنة ايضا.تم تحقيق الهدف من البحث عن طريق مناقشة اجرائية ومراجعة

بعض الادبيات ذات العلاقة. وفي النهاية تم ادراج عدد من من النقاط التي تعتبر كاستنتاجات التي تلخص

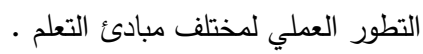

\section{Introduction}

It is difficult to define learning in a satisfactory manner, especially when it is as broad and abstract as the learning. It is likely that all those who search in to the meaning of learning

*Email: nada.hussien@sadiq.edu.iq 
carry with them some thoughts of what learning is. Without at least clear sense of what learning is, there would be no reason to devote time and effort for studying it. Finding explicit definitional issues can thus help avoid misunderstandings and facilitate communication among learning researchers. Most textbook definitions of learning describe it as a change in behavior that is due to experience, this can be considered a very basic definition of learning, in other words, learning is seen as a mapping function that maps gained experience onto behavior. One definition of learning is the effect of experience on behavior [1].A number of theories of learning have appeared like "lifelong learning", "informal and non-formal learning", "digital learning", "work place learning", "blended learning", "cooperative learning", "responsibility for his own learning" and "facilitation of learning", all these theories has made the formal definition of learning very complex or on what is demanded of a definition of learning, Nevertheless Learning theories help us to understand learning as a phenomenon. Some researchers try to come up with one comprehensive definition of what learning is and others focus on one concept or theory [2]. Researchers found that there are five categories were sufficient to describe the different views of learning to provide a general map of the qualitatively different ways in which the learning phenomenon is understood, as follow [3]:

1. Learning as the increase of knowledge.

2. Learning as memorizing.

3. Learning as the acquisition of facts, procedures etcetera which can be retained and/or utilized in practice.

4. Learning as the abstraction of meaning.

5. Learning as an interpretative process aimed at the understanding of reality.

In the mid of the last century, a new term in learning appeared, this term is machine learning, in this type of learning the aim is to make the machine act and behave like human to simulate the machine learn.

Machine learning is a branch of artificial intelligence that aims for giving the ability to machines to perform different jobs successfully by using intelligent software [4].

After the machine learning approach has evolved, a new approach in AI has appeared called deep learning. Deep learning is considered as a branch of machine learning that has its origins in mathematics, neuroscience and computer science [5].

In the last period, a new trend in learning has evolved named micro-learning, which is referred to as "bite-sized" because the entire learning process is presented in small chunks [6]. Micro-learning is becoming more interesting because it's way of learning and teaching the content in a relatively small and very specific burst [7].

\section{The problem statement and contribution}

Since learning is a broad term that can be a part of several topics. In this work a description of several terminology of learning is described and explained with clarification of the difference and similarity among different types of learning terms. The paper take into consideration three techniques of learning two of them are bran new techniques which are deep learning and micro-learning.

\section{Methodology}

Learning computation refers to any change that is made for the computing system that allow it to accomplish the same task(s) or another task(s) in better way from the same single or population based.

\subsection{Machine Learning}

The statistical learning methods are considered as the back bone to develop machine intelligence. Because the algorithms of machine learning require data to learn, there are familiar terms concerned with data such as Knowledge Discovery from Data (KDD), pattern recognition and data mining. Machine learning algorithms aims to write programs for 
computers that will make them learn and enable them to perform different tasks, such as prediction. The goal of learning is to construct a model that takes a specific input and produces the desired output. Sometimes, the model is understandable, whereas, in some other times, it can be like a black box for us, the working of which cannot be explicitly explained. Such model can mimic what the machine is built for [4].machine learning is applied to make the machine handle data more effectively, sometimes it's hard to extract patterns and information from raw data by using machine learning the machine will learn from such data[8]. To achieve the learning process, machines are trained using different statistical models and algorithms to analyze data. The data are usually identified by characteristics that are measurable called features and a ML algorithm

try to find a correlation between output values called labels and the features. Then, all the information gained from the training stage is used to make decisions or recognize patterns based on new data. ML is useful for problems such as classification, regression, association rules determination and clustering. Depending on the style of the learning, ML algorithms are classified into four categories[5]:

Supervised Learning: Supervised learning handles issues that involve regression like estimating life experience, weather population growth prediction and forecasting, by using various algorithms like Random Forest or Linear Regression. Supervised learning is used to classify problems like speech recognition, digit recognition, identity fraud detection and diagnostics, by employing algorithms such as Support Vector Machines, Random Forest, Nearest Neighbor and others. Supervised learning involves two phases, testing phase and training phase. The training phase uses data sets that have known labels. The machine learning algorithms works to learn the relationship between the values of the input from the training dataset and labels for each input and then tries on the testing data to predict the output[5].

Unsupervised Learning: Unsupervised learning handles issues such as dimensionality reduction and visualization used for feature extraction, big data, or the discovering hidden patterns or structures. Moreover, unsupervised learning is best can be very useful for clustering problems such as, recommendation systems, customer segmentation and targeted marketing. Unlike supervised learning, the labels are not needed or available. Algorithms in this type try to identify patterns founded on testing data and then cluster these data according to these patterns or predict future values[5].

Semi-supervised Learning: This is a combination of supervised and unsupervised learning. Both types of data are used labeled and unlabeled. It mostly works like the unsupervised learning with partial use of labeled data, it is considered as an improvement on unsupervised learning[5].

Reinforcement Learning: In this learning type, the algorithms use tuning parameters for prediction of the output for a problem. Then, the predicted output will be used as an input parameter for the algorithm and new output is computed until finding the optimal output. Reinforcement learning is used for applications like skill acquisition, AI gaming, real-time decisions and robot navigation [5].

\subsection{Deep Learning}

Deep networks is able to learn from data in the same way that babies are able to learn from the world around them, starting with fresh eye sight and gradually acquiring more skills needed to navigate environments around them. The origin of deep learning approach goes back to the beginning of artificial intelligence in the $1950 \mathrm{~s}$, when there were two visions that compete for how to create an AI: the first vision was based on computer programs and logic, which dominated the field of AI for decades; while the second vision was based on learning from data directly, which took much longer time to mature. In the 20th century, when computers were poor and data storage was costly by today's standards, logic was the most 
efficient way to solve computing problems. Skilled programmers for each problem wrote a different program and the bigger the problem they are dealing with lead to bigger program. Today computer power that is available and big data is numerous and it is faster solving computing problems using learning algorithms that are more efficient and more accurate. Many difficult problems can be solved using the same learning algorithm; its solutions need much less work than writing a different program for each problem. The Deep Learning Revolution has two convoluted themes: how artificial intelligence evolved and how human intelligence is evolving. The difference between the two types of intelligence is the time needed for evolving, human intelligence took many millions of years to evolve, but AI is evolving faster on a trajectory measured in decades. The conversion from AI based on logic, symbols and rules to deep learning approach based on learning algorithms and big data was not easy [9].

Deep learning in recent years has proven to be accurate on some tasks that surpass that of a human. Actually, the recent results gained from deep learning algorithms that transcend human ability and performance in image recognition tasks that can't likely considered by computer vision experts in the last decade. Many architectures of deep learning that presents such phenomenal performance are not a results of a random connections of computational units. The outstanding performance shown by deep neural networks reflect the fact that biological neural networks obtained much of their strength and power also from depth. Furthermore, it is not fully understood how biological networks are connected. In the cases that the biological network structure is understood at some grade, great achievements have been reached by modeling artificial neural networks based on those networks [10].Figure(1) shows the ability of deep learning to classify in a very detailed manner.

The main goal in applying deep learning to CV (computer vision) is to remove the exhausting, and limiting, feature selection process. Deep neural networks are very efficient for this process because it works in layers and each layer of a neural network is responsible for building up features and learning to represent the receives input[11].

The architecture of deep-learning is a like stack of modules that is considered as multilayer, all of these models or most of them are undergo to learning, all or (many) of them process non-linear input-output mappings. In this stack each module divert its input to boost both the invariance and selectivity of the representation of the model. With several layers that are nonlinear, say a depth of 5 to 20 , the system will be able to implement extremely complex functions of its inputs that are sensitive to details - the system can distinguishing a dog from a muffin - and incurious to variations that are irrelevant such as the pose, background, surrounding objects and lighting[12].

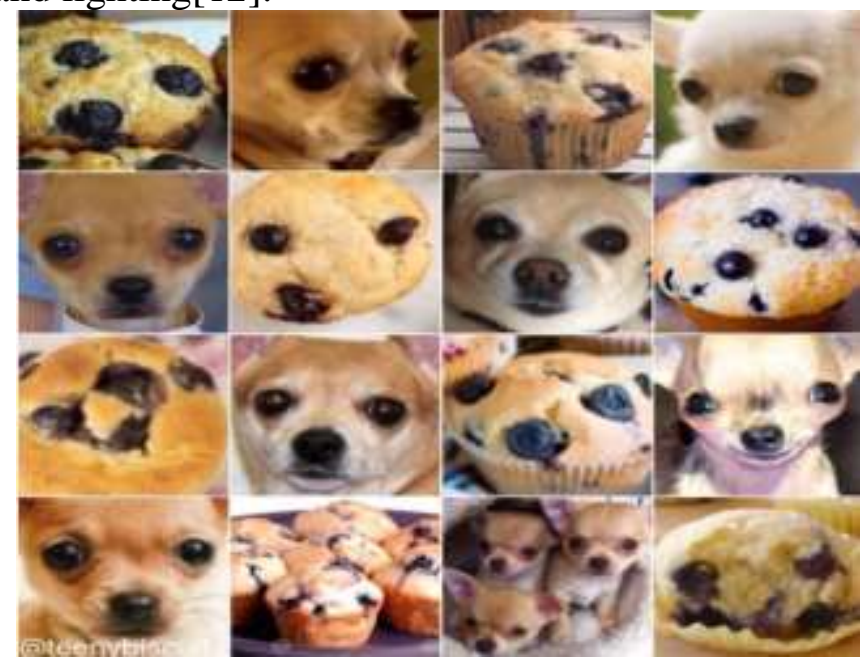

Figure 1-deep learning classification ability to distinguish a muffin from a dog 


\subsection{Micro-Learning}

Micro-learning presents the opportunity to absorb and retain the information provided and activities that are more digestible and manageable more easily. The way micro-learning identifies small portions of learning content that consists of fine-grained, loosely-coupled which are interconnected and shortened learning activities, define the concentrate on the individual learning needs.

Micro-content and micro-learning together determine how to submit a quantum of information and knowledge, structured in many short sections, fine-grained, interconnected and well-defined. The piece of information whose size is determined by a single topic, content that covers a single concept or idea and can be accessed via a single URL, being suitable for using in handheld devices, web-browsers, emails all that are refers to Microcontent. Thus, micro-content is the part that merges into micro-learning [6].

In micro-learning knowledge are acquired using instructional design techniques, abilities and skills which happen on a daily basis. The way that micro-learning works is by taking information naturally by learner's brain, so that the body and brain does not get stressed. One of the essential features of micro-learning that works saliently is that it allows the learner to find what he or she is looking for exactly. It enables the learner's brain to explore and satisfy its own patterns and its own curiosity [7].

\section{Literature Survey}

Here a description survey for the more important and usage nowadays for practical applications using different learning terms (machine learning, deep learning and microlearning).This section is portioned into four su0062sections according to the learning type as illustrated

\subsection{General Learning}

A. Qvortrup, et al. in 2016[2], mentioned in their publication different definitions and theories about learning and how learning theory consists of the student learning and the behavior of the teacher and they also explained how to construct boarders and moving between teaching activities, learning objectives and different students learning methods through participating, and how to link between methods of education and methods of learning.

de Houwer and Moors, in 2014[1], stated in their paper a comprehensive definitions about the term learning by giving a detailed functional definition that might solve the issues in the alternative definition by assuming that learning is a change in the behavior of the learner.

van Rossum and Hamer in 2010[3], presented in their publication a model of linked learning that consists of 6 stages that can help the student in learning and teaching, these stages consist of complex structure in which each stage depend on another .

M.G.Behlol, et al in 2010[13] stated in their paper a comparative study between behaviourists, connectivists and humanists in regards to their opinions in learning, the authors listed the differences and the similarity between the approaches. The authors reaches to different definitions to what learning is, a fact memorizing, an increase of knowledge , meaning abstraction, skills gaining or adaptation and improvement.

\subsection{Machin Learning}

H. S. Abdullah and H. D. Hasan in 2019[14] proposed in their paper a system to classify documents, in this work the authors used machine learning for classifying the documents into their suitable class depending on the textual content of the document, using feature extraction then feature selection and by modified naïve bays classifier the proposed system were able to reach $95 \%$ accuracy of correctly classified documents.

H. S. Abdullah and A. H. Jasim in 2016 [15] stated in their work the use of machine learning for image segmentation by employing ant colony algorithm, taking advantage of the parallelism nature of the ant colonies to find the best segment in less computation time than using the single core ant colony algorithm at the end the segments found by all the ant 
colonies is combined to form the final segmentation. The proposed technique perform better than the traditional sequential algorithm with the same precision in segmentation results.

Michael Luckertmoritz in 2015[16], in his work stated that using machine learning can improve the quality of document translation by using knowledge discovery process consisting of several phases, gathering and preprocessing data, choosing an appropriate data mining technique to find patterns among data in dataset and interpreting them. Using five different approaches of machine learning showed a prediction accuracy of $72: 24 \%$ for the binary classification.

Zur Erlangung in 2010[17], stated in his work that machine learning can be used in technology enhanced learning to extract practice knowledge. Practice knowledge is extracted from observational data and then the model of practice knowledge are applied to the learner context form support. Two subclasses are presented in this work, one for general feedback and the other for detailed feedback. In the first one a supervised machine learning approach is used while the second one will provide in depth information for the leaner.

\subsection{Deep Learning}

N.E. Sahla in 2018[18], reported in the publication that implementing convolution neural network $(\mathrm{CNN})$ used in deep learning for classification can give good result when it is used with smart picking strategy where a robot tries to classify object by swapping the front of the object to the classification probability vector. This ensure minimal to no error cost and faster picking (classification).

A. B. Fakultät in 2017[19], stated in his work that using deep learning,(CNN) and a view point preprocessing extraction enhanced the recognition process and outperform the other neural networks models.

Alina Kloss in 2015[20], presented in her work a model for object detection using deep convolution neural network based on eye tracking data technique recorded form 15particepaint. The deep convolution neural network is used to extract features form the input image that are used in constructing saliency map. This map is used to provide information about the region of interest within an image to reduce the preprocessing by reducing the part of the image to search in to detect target object.

\subsection{Micro-Learning}

Christina Drakidou in 2018[21], stated in her work that using micro-learning in e- learning courses enhance the long life learning and continuous learning. The author implanted several example courses that are carefully designed, supervised and implemented by well-trained instructors-facilitators. The author proved that micro-learning can be used as an E-learning technique that will improve learning outcomes.

G. S. Mohammed et al. in 2018,[22] reported in their work that an important requirement for successful learning is experiencing learning activities on a regular basis and keeping it memorable for long time. Micro-learning can be delivered in small chunks which make memorable and easy to understand the authors test micro-learning technique on primary school student and they found that student which learned using micro-learning gained better learning than student that were subjected to traditional learning.

Elaine Rettger in 2017[23] in her work presented the idea of employing micro-learning using mobile devices for academic studies and how the delivery of instruction- distributed presentation will affect the learning outcome and the author proved that students receiving small units of instruction and information over a series of days would perform much better than students receiving the instruction and information in a massed unit.

Norm Friesen in 2007[24], suggested in his work that the traditional learning is forcing constrains on the learner. Micro-learning is giving the ability for personalized learning and freeing the learner from those constrains. The author thinks that these features of microlearning are important and valuable. 


\section{Discussion}

In this section a brief discussion about each work is presented

de Houwer and Moors, in 2014[1], the researchers presented functional definition of learning that will solve the problems related to other definition and it also have advantages such as cognitive learning psychology. This definition also has its down sides such as difficult to verify and cognitive researchers that deal with this type of definition will have change their habitual conceptualization of learning into mental mechanism. Changing such habits of researchers will take effort and time might thus meet resistance.

A. Qvortrup, et al. in 2016[2], the researchers presented different definition to the phenomena of learning. These definition embrace different aspects, theories and concepts of learning as student and teacher behavior. This publication aims to focus on several viewpoint of number of researcher and how to improve those viewpoint about learning.

van Rossum and Hamer in 2010[3], the authors presented a six stage model of linked learning this model has its advantages like motivation, learner sense of control over what is to be learned and how, expectations towards the learning and teaching environment.

M.G.Behlol, et al in 2010[13] the researchers presented a comparative study between different approaches of learning concept in the views of behaviourists, connectivists and humanists the study contributed in helping teachers and students to boost the learning process by better understanding what learning means and how accomplish the concept in their journey of learning and teaching.

H. S. Abdullah and H. D. Hasan in 2019[14] the authors proposed a model for document classification based on textual information. The model composed of several stages, first preprocessing, the second step feature extraction, the third step is a proposed feature selection that select only one feature for each document and finally classification using modified naïve bays classifier. The model were tested on two data set and the results were on average $95 \%$ of precision, recall and $\mathrm{F}$ measure for both datasets.

H. S. Abdullah and A. H. Jasim in 2016 [15], the authors used ant colony algorithm for document segmentation taking advantage of the parallelism nature of the ant colony algorithms. Each colony will segment the document and the results of all the colonies will companied together to form the best segmentation results. The proposed segmentation technique reduces the computation time considerably.

Michael Luckertmoritz in 2015[16], the author presented an approach for document translation using five machine learning techniques. K-Nearest Neighbor classifier scored the maximum results $72: 24 \%$ accuracy when having access to the original document and $62: 93 \%$ accuracy without access to it. When having access to the document the results showed zero Misclassification while 14:29\% when having no access. The work also has its limitation, some of machine learning approaches did not optimize as desired for the problem as it should be due to computation resource limitation. Another limitation to this work is the data extraction process, which has been extracted from PDF documents causing some errors during the process a suggestion to this problem is using XML files.

Zur Erlangung in 2010[17], a machine learning technique is used to capture practice knowledge to support the learner. This practice knowledge can be found in both, Detailed, and General Feedback Machine Teaching. Machine Teaching assume the knowledge is to be observable and therefore it can adds another layer of knowledge to Technology Enhanced Learning compared to traditional Technology Enhanced Learning methods and approaches. This approach is able to compute meaningful feedback to the learner that will help and support him. This approach also has its limitation in the context of user interface, features for the detailed feedback and matrix factorization that can be improved to give butter results.

N.E. Sahla in 2018[18], the author presented an approach for object classification using CNN which gave accuracy $44.64 \%$ butter results than traditional ANN. Implementing CNN needs 
more work to do like using GPU instead of depending on just CPU and it needs a huge dataset to overcome overfitting problem, but when $\mathrm{CNN}$ is implemented in the right way scalability is not a problem and a cloud- based can be used to decrease the training time needed.

A. B. Fakultät in 2017[19], the author used CNN for galaxy image classification with view point extraction as a preprocessing. The proposed approach were able to highlight the morphological features of the galaxy images by enhancing and isolating different object for different views. A filter reduction process were used for computational time reduction. This approach was able to achieve RMSE of 0.082 for the validation dataset and 0.084 for the training dataset.

Alina Kloss in 2015[20], the researcher proposed an approach for reducing time and region of interest for object detection problem using $\mathrm{CNN}$ and saliency map. The $\mathrm{CNN}$ is used to extract features while the saliency map use these features to reduce the time for object detection by reducing the region of interest to be searched. The results showed that this method reduced time and needed processing for object detection however the method can further be improved for better results.

Christina Drakidou in 2018[21],the author use's micro-learning for enhancing e- learning and to contribute in long life and continuous learning by improving on demand and on sit learning for the learner. Micro-learning proved its flexibility and adaptability to deliver micro-content using easy to access techniques like email, mobile and network social society. Using microcontent make it easy to update and it can considered as standalone learning units though can be used as supporting units in other learning techniques. The researcher found that using micro-learning can improve the e-learning and can be very helpful for the people who are seeking continuous learning.

G. S. Mohammed et al. in 2018[22], the researchers employed micro-learning as a technique to improve traditional learning. The researchers used two groups of primary students as case studies, the results showed that the group who received micro-learning showed better learning than the other group by $18 \%$. The proposal proved that micro-learning can improve learning ability as well as aiding their long term memory.

Elaine Rettger in 2017[23], the author presented the idea of implementing distributed presentation micro-learning to deliver micro-content using mobile devices. The study consist of four conditions and with the participation of 311 student in higher education, 173 completed all four conditions. The results of this study showed that there were no significant effect on the delivery of the content as instruction but it had a good effect on the testing in a one way and $2 * 2$ factorial design. A positive response were collected from student receiving micro- instruction on their mobile device.

Norm Friesen in 2007[24], the researcher presented the idea of personalizing the learning through micro-learning and freeing the learner from traditional learning constraint. The researcher also mentioned that micro-learning is the present learning and the traditional learning is the history of learning.

After discussing several works on each type of learning a brief comparison among them is presented in table (1). 
Table 1-comparison among learning types

\begin{tabular}{|c|c|c|c|}
\hline & Machine Learning & Deep Learning & Micro-learning \\
\hline Idea & $\begin{array}{l}\text { Train the machine to make it Learn, } \\
\text { act and make decision like human }\end{array}$ & $\begin{array}{c}\text { train neural } \\
\text { network to find } \\
\text { detailed information }\end{array}$ & $\begin{array}{l}\text { Using micro- } \\
\text { content to deliver } \\
\text { memorable and } \\
\text { long life learning }\end{array}$ \\
\hline Applications & $\begin{array}{c}\text { Classification/clustering/recognition } \\
\text { Path planning/ feature extraction/ } \\
\text { optimal solution search }\end{array}$ & $\begin{array}{l}\text { Classification/ } \\
\text { recognition/ feature } \\
\text { extraction }\end{array}$ & $\begin{array}{c}\text { e-learning/micro-content } \\
\text { delivery/ } \\
\text { mobile instruction } \\
\text { delivery }\end{array}$ \\
\hline $\begin{array}{l}\text { Techniques } \\
\text { used }\end{array}$ & $\begin{array}{l}\text { ANN/ GA/ Swarm intelligence/ Data } \\
\text { mining }\end{array}$ & $\begin{array}{l}\text { CNN/ Recurrent } \\
\text { Neural Network } \\
\text { (RNN) }\end{array}$ & Micro-content \\
\hline $\begin{array}{c}\text { Time } \\
\text { consuming }\end{array}$ & Acceptable range & High & Low \\
\hline $\begin{array}{l}\text { Computational } \\
\text { resources } \\
\text { consuming } \\
\end{array}$ & Manageable & High & low \\
\hline Used dataset & $\begin{array}{c}\text { Information type: } \\
\text { \{images, text, videos }\} \\
\text { Size: medium to huge } \\
\text { Specificity: Homogenous } \\
\text { information }\end{array}$ & $\begin{array}{c}\text { Information type: } \\
\text { \{Images,text,videos }\} \\
\text { Size: huge } \\
\text { Specificity: } \\
\text { Homogenous } \\
\text { information } \\
\end{array}$ & $\begin{array}{c}\text { Information type: } \\
\{\text { Images,text,videos } \\
\text { Size: small } \\
\text { Specificity:Heterogeneous } \\
\text { information }\end{array}$ \\
\hline
\end{tabular}

From table (1), it can be seen that the comparison is illustrated as procedural way, this means that the compared methods are distinguished according to the behavior and performance for each one, this because of there are no obtained specific points to make a practical comparison due to the following reasons:

- The micro-learning is still a mellow technique, in other words it is not used as a machine learning yet as a practical applications.

- The limitations of deep learning applications (in a specific manners), make it a particular technique as well as the evaluation of the resulted view.

\section{Conclusions}

Learning concepts are evolving very fast through the decades, starting from general learning into machine learning and then into deep learning and finally micro-learning. Machine learning approach appeared in the fifties with a lot of potentials dealing with giving the ability to machine for learning and make intelligent decisions, which make the machine learning to achieve the main goal of it which is making change in system behavior to adapt the performance of it, in other words any change in the system which guarantee success training that lead to proper learning.

While deep learning is about how to make the machine recognizes and classifies different objects in a very detailed manner, making the machine tail the difference between a Chihuahua dog or muffin as shown in figure(1). Although deep learning has the ability to make really fine classification and recognition task, it need a huge dataset to overcome the overfitting problem, it consumes a lot of time and computational resources.

The latest concept appeared in learning is micro-learning, it is simple easy to implement technique that gives good results in terms of memorable and long life learning that is delivered as chunks of small size information, it assures the continuity of learning in the long term. Micro-learning also has downside regarding its acceptability in society to depend on.

\section{References}


[1] Jan De Houwer and Agnes Moors, January 2013, "What is learning? On the nature and merits of a functional definition of learning", Psychonomic Bulletin \& Review. DOI: 10.3758/s13423-0130386-3

[2] Ane Qvortrup, Merete Wiberg,Gerd Christensen \& Mikala Hansbøl, 2016,"On the Definition of Learning", Denmark ,University Press of Southern Denmark.

[3] Erik Jan van Rossum and Rebecca Hamer, 2010, "The Meaning of Learning and Knowing", Netherland, SENSE Publishers Rotterdam/Boston/Taipei.

[4] Mohssen M. Z. E. Mohammed, Muhammad Badruddin Khan and Eihab Bashier Mohammed Bashier, 2017, "Machine Learning: Algorithms and Applications", Taylor \& Francis Group, LLC, DOI: $10.1201 / 9781315371658$.

[5] Terrence J. Sejnowski, 2018, "The Deep Learning Revolution”, The MIT Press ,Cambridge, Massachusetts London, England.

[6] Luminiţa Giurgiu, 2017, "Microlearning an Evolving Elearning Trend", Scientific Bulletin Vol. XXII No 1(43), DOI: 10.1515/bsaft-2017-0003.

[7] Omer Jomah, Amamer Khalil Masoud, Xavier Patrick Kishore and Sagaya Aurelia, March 2016, "Micro Learning: A Modernized Education System", BRAIN. Broad Research in Artificial Intelligence and Neuroscience Volume 7, Issue1, https://www.semanticscholar.org/paper/MicroLearning\%3A-A-Modernized-Education-System-JomahMasoud/f8311fe4c784518d99c41e383e859dacc8c3220c

[8] Ayon Dey, 2016, "Machine Learning Algorithms: A Review" International Journal of Computer Science and Information Technologies, Vol. 7 (3), 1174-1179. https://www.ijsr.net/archive/v9i1/ART20203995.pdf

[9] Fotios Zantalis, Grigorios Koulouras, Sotiris Karabetsos and Dionisis Kandris, 2019 ," A Review of Machine Learning and IoT in Smart Transportation", Future Internet,doi:10.3390/fi11040094.

[10] Charu C. Aggarwal, 2018, " Neural Networks and Deep Learning", Springer International Publishing AG, part of Springer Nature, EBook.

[11] Nikhil Buduma, Nicholas Lacascio, june 2017" Fundamentals of Deep Learning Designing NextGeneration Machine Intelligence Algorithms", first addition ,USA, Oreilly.

[12] Yann LeCun1,2, Yoshua Bengio3 \& Geoffrey Hinton, 2015 "Deep learning Review", Macmillan Publishers Limited, doi:10.1038/nature14539.

[13] Dr. Malik Ghulam Behlol, Dr. Hukam ,Dad , 2010 “Concept of Learning”, International Journal of Psychological Studies.

[14] Hasanen S. Abdullah , Hala Dhiaa Hasan, 2019 " Intelligent Documents Classification System" , Al-Mansour Journal/ Issue (31), https://muc.edu.iq/oldwebsite/mucj/31/english/e9-a31.pdf

[15] Hasanen S. Abdullah, Ammar H. Jasim" Document Image Segmentation using Multi ANT Colonies Algorithm (MAC) on a Multi-Core Processor",2016, International Journal of Computer Science and Mobile Computing, https://www.researchgate.net/publication/312222705

[16] Michael Luckert , 2015, " Using Machine Learning Methods for Evaluating the Quality of Technical Documents", M.S.c. thesis, Linnaeus University, computer sciencedepartment.

[17] Zur Erlangung, 2010," Machine Teaching A Machine Learning Approach to Technology Enhanced Learning",M.S.c thesis, university of Technischen Universit"at Darmstadt.

[18] N.E. Sahla. , 2018," A Deep Learning Prediction Model for Object Classification”, M.S.c. thesis, Delft university of Technology.

[19] Andriy Borovkov, 2017, "Image Classification with Deep Learning”, M.S.c. thesis,university of Hamburg.

[20] Alina Kloss, 2015,“Object Detection Using Deep Learning -Learning where to search using visual attention", M.S.c. thesis, Eberhard Karls University Tuubingen.

[21] Christina Drakidou, 2018, "Micro-learning as an Alternative in Lifelong eLearning", MA Dissertation, Aristotle University of Thessaloniki School of Italian Language and Literature.

[22] Gona Sirwan Mohammed, Karzan Wakil and Sarkhell Sirwan Nawroly, 2018, "The Effectiveness of Microlearning to Improve Students' Learning Ability", International Journal of Educational Research Review, DOI: 10.24331/ijere.415824.

[23] Elaine Rettger, 2017, "Microlearning with Mobile Devices: Effects of Distributed Presentation Learning and the Testing Effect on Mobile Devices",Ph.D. Dissertation, Arizona State University. 
[24] Norm Friesen,, 2007, "The Microlearning Agenda in the Age of Educational Media", Thompson Rivers University,Canada.

[25] https://www.researchgate.net/publication/255582537_Outline_of_a_Microlearning_Agenda 